\title{
The Contribution of a Body Scan Mindfulness Meditation to Effectiveness of Internet-Delivered CBT for Insomnia in Adolescents
}

\author{
Eduard J. de Bruin ${ }^{1,2}$ (D) AnneMarie Meijer ${ }^{2} \cdot$ Susan M. Bögels ${ }^{2}$ \\ Published online: 17 January 2020 \\ (C) The Author(s) 2020
}

\begin{abstract}
Objectives Insomnia is highly prevalent among adolescents, with severe consequences for daily life, including mental disorders. Cognitive behavioral therapy for insomnia (CBTI) can be effective in internet-delivered and face-to-face modalities. However, it is yet unclear what the contribution is of mindfulness-based techniques to the effectiveness of traditional CBTI. The present study investigated whether use of a body scan mindfulness meditation influenced the effectiveness of CBTI for adolescents.

Methods Adolescents who had received 6 weeks of internet CBTI were divided into a group who had practiced a body scan meditation $(N=26$, age $M=15.33$ years, $\mathrm{SD}=1.42,77 \%$ girls) and a group who had not $(N=28$, age $M=15.64$ years, $\mathrm{SD}=$ $1.80,82 \%$ girls). Differences between the groups were analyzed for subjective and objective sleep outcomes from baseline to post-treatment and to 2-month follow-up.

Results Moderate to large effect size improvements appeared for sleep parameters from 7-day actigraphy and sleep logs, and large effect size decreases of self-reported symptoms of insomnia and chronic sleep reduction, at post-treatment for both groups. Improvements were maintained at follow-up. However, where the non-body scan group showed no significant change of wake after sleep onset from actigraphy, and self-reported shortness of sleep and irritation, there were medium effect size improvements of these outcomes in the body scan group.

Conclusions These results indicate that the use of a body scan mindfulness meditation in CBTI may have an additional positive effect on sleep, above and beyond traditional CBTI techniques.
\end{abstract}

Keywords Cognitive behavioral therapy for insomnia $\cdot$ Adolescents $\cdot$ Internet interventions $\cdot$ Body scan $\cdot$ Mindfulness

Insomnia is one of the most common sleep disorders among adolescents, with point-prevalence rates ranging from 7.8 up to $23.8 \%$ (Dohnt et al. 2012; Hysing et al. 2013). Moreover, meta-analyses of mental disorders worldwide indicate that insomnia could well be one of the most prevalent mental health problems in adolescence (Polanczyk et al. 2015; Wittchen et al. 2011). Chronic sleep problems such as insomnia can cause or exacerbate other mental problems and disorders in a

Eduard J. de Bruin

E.J.deBruin@utwente.nl

1 Centre for eHealth and Wellbeing research, Department of Psychology, Health and Technology, University of Twente, P.O. Box 217, 7500 AE, Enschede, The Netherlands

2 Research Institue of Child Development and Education, University of Amsterdam, Amsterdam, The Netherlands bidirectional fashion (e.g., Gregory and Sadeh 2012; Kahn et al. 2013; Sivertsen et al. 2012). Relations have been shown between insomnia and depression (Roberts and Duong 2013), anxiety (Johnson et al. 2006), substance abuse (Bootzin and Stevens 2005), inattention (Chervin et al. 1997; Dahl and Lewin 2002), attention deficit hyperactivity disorder (ADHD; Owens et al. 2013), conduct problems (Meijer et al. 2010), and somatic health problems (Dahl and Lewin, 2002; Zhang et al. 2011).

For adults, treatment with cognitive behavioral therapy for insomnia (CBTI) has been shown to be effective in many modalities over the past two decades, including face-to-face individual CBTI, group CBTI, and internet-delivered CBTI (Espie et al. 2012; Lancee et al. 2012; Medalie and Cifu 2017; Morin et al. 2006; Van Straten et al. 2018). In a recent study into the efficacy of CBTI for adolescent insomnia, we found comparable outcomes for face-to-face group therapy and guided individual internet therapy (De Bruin et al. 
2015). Generally, CBTI consists of stimulus control therapy, cognitive therapy, relaxation training, and sleep restriction, combined with sleep hygiene and psycho-education. With the emergence of mindfulness-based interventions (MBI) additional or combined mindfulness-based and cognitive behavioral techniques have been developed to treat insomnia (e.g., Ong et al. 2014; Wong et al. 2017). A recent study by Blake et al. (2016) of MBIs targeted at adolescents with elevated levels of sleep problems and anxiety symptoms showed improvements of objective and subjective sleep outcomes.

However, although the literature on adolescent CBTI is slowly increasing, it lags behind when compared with adult CBTI regarding both theoretical underpinnings and working mechanisms that can further the development of insomnia treatment for adolescents specifically. The multifaceted nature of insomnia is represented in several models on the causation and perpetuation of insomnia, such as the 3-P model, with predisposing, precipitating, and perpetuating factors (Spielman et al. 1987); the neurocognitive model on attenuation of mesograde amnesia of wakefulness (Perlis et al. 1997); and the psychobiological inhibition model on the failure to inhibit wakefulness, and selective attending to life stressors and insomnia symptoms (Espie et al. 2006). In these models the interaction between thoughts and physiological state (e.g., psychophysiological hyperarousal) plays an important role, with subtle differences between cause and effect. For instance, according to the psychobiological model, cognitive processes such as worry and attending to not sleeping are regarded as perpetuating factors of insomnia, whereas in the neurocognitive model these cognitive processes are not central to insomnia, but secondary (i.e., one is awake because of worry, versus one is worrying because one is awake) (Perlis et al. 2010). Research on mechanisms and mediators of adult CBTI has provided support for most of the theoretical factors of insomnia (Schwartz and Carney 2012). Regarding hyperarousal, the evidence suggests that adult primary insomnia is associated with inappropriate psychophysiological arousal as shown by increased high-frequency EEG activation, abnormal hormone secretion such as increased evening and early night cortisol secretion as an indicator of the activity of the HPA (hypothalamic-pituitary-adrenal) axis, increased metabolic activation, elevated heart rate, and increased sympathetic nervous system activation during sleep (Bonnet and Arand 2010; Riemann et al., 2010). Findings in the adolescent population indicate that the above mentioned cognitive processes are important in the perpetuation or amelioration of adolescent insomnia as well (De Bruin et al. 2014b; Fernandez-Mendoza et al., 2016), but little is known about which facets of insomnia can be affected by interventions that target these processes in adolescents.

In the cognitive behavioral framework for treating insomnia, the techniques that aim at affecting psychophysiological arousal are cognitive therapy and relaxation training
(Morgenthaler et al. 2006; Schwartz and Carney 2012). People with insomnia can develop apprehension about bedtime and the inability to fall asleep. In adolescents, this apprehension can result in dysfunctional beliefs such as "I have to sleep now otherwise I won't be able to do well at my exam tomorrow" or "I need at least 9 hours of sleep", which in turn can lead to psychophysiological hyperarousal (Palagini et al. 2017). Hyperarousal that results from worry or rumination before or during the sleep period can result in chronic, somatized tension and positive conditioning of maladaptive behaviors such as delaying bedtimes and associating the bed and bedroom with being awake (Hauri and Fischer, 1986; Perlis et al. 2010; Riemann et al., 2010). By systematically challenging the dysfunctional cognitions, and where appropriate replacing them by more functional thoughts, cognitive therapy for insomnia aims to decrease psychophysiological hyperarousal. Relaxation exercises such as progressive muscular relaxation in CBTI are used to deliberately promote relaxation by sequentially tensing and relaxing muscles in the body (Morgenthaler et al. 2006). One exercise from MBIs that could be especially relevant for this psychophysiological arousal facet of insomnia is the body scan. The aim of the body scan is to emphasize awareness of current physical sensations by directing non-judgmental attention to each part of the body (Thompson and Gauntlett-Gilbert 2008), thus affecting both the physical arousal that is caused by worry, and the worry itself, by affecting the process that ties these two together. The non-judgmental awareness to body parts and physical sensations while lying down may benefit an adolescent with insomnia in three ways. First, it may decrease physiological arousal by prolonged awareness of a calm respiration. Second, cognitive arousal may decrease by engaging the hyperactive mind in focusing on the body. And third, it may have a paradoxical effect, because the instruction is not to fall asleep but to lie down without moving.

Moreover, the non-judgmental nature of the attention seems especially salient in light of the attentional bias that may arise in insomnia. Espie et al. (2006) proposed that sleep effort-which may emerge when automaticity of sleep is disrupted or inhibited by selectively attending to sleep - is a key perpetuating factor of insomnia. Furthermore, considering that most adolescents are not familiar with meditative practices, this lying down practice of mindfulness has the added benefit that it may be more comfortable in the earlier stages of meditation practice, and it could facilitate being still for longer periods of time (Kabat-Zinn 2016), a notion that is underscored by the finding that the body scan is among the most popular meditation practices from Mindfulness-Based Stress Reduction (MBSR) in novel practitioners (Ribeiro et al., 2018).

Summarizing, insomnia is highly prevalent among adolescents and can have severe consequences for daily life, including mental problems and disorders. Recently, several studies 
have indicated that treatment for adolescent insomnia can be effective in internet-delivered and face-to-face formats and that mindfulness-based techniques could enhance effectiveness and tailor treatment for adolescents specifically. However, it is yet unclear what the contribution is of mindfulness exercises to the effectiveness of traditional CBTI. In the present study, we therefore investigated whether practice of a body scan mindfulness meditation influences effectiveness of CBTI for adolescents.

\section{Methods}

\section{Participants}

We used the data from two subsamples with a total of 56 adolescents of which two were excluded because of lack of registration of the body scan use. That brought the total to 54 adolescents with insomnia who received internet-delivered CBTI in a larger study on effectiveness of CBTI for adolescents, of which the main results have been published elsewhere (De Bruin et al. 2014a; De Bruin et al. 2015a; De Bruin 2015b; De Bruin et al. 2018). Recruitment was conducted with (electronic) newsletters and websites for mental health care professionals, schools, and general physicians, and through the national news media. Inclusion criteria were (1) age 12-19 years and (2) insomnia disorder according to the Diagnostic and Statistical Manual of Mental Disorders-Fifth Edition (DSM-5; American Psychiatric Association, 2013). Exclusion criteria were (1) suicidal intentions and drug abuse, screened by clinical scores and item screening on the Youth Self Report (YSR; Achenbach 1991) and from a 1-h intake interview; (2) other sleep disorders than insomnia (e.g., apnea, delayed sleep wake phase disorder); (3) a diagnosis of other mental disorders (e.g., depression, ADHD), or presently being treated for psychological or sleep problems; and (4) use of drugs or medication that interfere with sleep.

\section{Procedures}

This study was part of a larger study of effectiveness of CBTI for adolescents, which was approved by the medical ethical committee of the Academic Medical Center in Amsterdam and registered at the International Standard Randomized Controlled Trial Number Register (ISRCTN33922163). All participants were screened for inclusion and exclusion criteria, and when meeting the criteria, they were diagnosed in a $1-h$ diagnostic interview by a psychologist specialized in sleep medicine. After informed consent was obtained, participants in the present study were randomly assigned to internetdelivered CBTI.

Participants received six weekly sessions of internetdelivered CBTI. The protocol contained psychoeducation, sleep hygiene, restriction of time in bed, stimulus control, cognitive therapy, and a body scan meditation. Sessions were conducted by experienced and certified sleep psychotherapists. For each session, participants logged onto a website where at a fixed time of day, once every week, a session was made available with written exercises, movies, sound files, interactive questionnaires, automated feedback, and written feedback from a sleep therapist that included a personalized bedtime advise. After the second internet session, participants had a 15-min chat session with their personal therapist. Participants could post questions on their personal login page or in the daily sleep logs, which were responded to by the sleep therapist in the weekly, personalized feedback, or in the chat session. All sessions started with a review of the sleep variables from the sleep logs. Bedtime advice based on these sleep variables was explained, after which exercises of CBTI were introduced or continued from the previous sessions. The body scan meditation consisted of a 20-min auditory body scan instruction from a licensed MBSR/MBCT therapist and was introduced in session two. Participants could download the auditory file for use on electronic devises, or use it online. In sessions three to six, the body scan meditation was reviewed and further explained in terms of its possible effects on stress, arousal, and relaxation, and how these relate to sleep problems. In the automated and personalized feedback, the practice of the body scan meditation, and the other CBTI techniques, was encouraged, but participants could decide themselves whether or not to continue practicing the CBTI exercises. To clarify practice, CBTI exercises were modeled by an actor/actress, playing an adolescent with insomnia.

\section{Measures}

Measurements were obtained at baseline, directly after treatment (about 7 weeks after baseline), and at 2-month followup. Sleep parameters were obtained with sleep $\log _{s}$ and actigraphy for seven consecutive days at each measurement occasion. Questionnaires on symptoms of insomnia and chronic sleep reduction were obtained once each measurement occasion.

Sleep logs are considered to be a reliable source for measuring subjective self-reported sleep times (Carney et al. 2012). Sleep logs consisted of eight questions measuring bedtime, lights-out time, wake up and get up times, estimations of sleep onset latency (the time between lights-out and sleep onset), and wake after sleep onset (time awake at night after the first sleep onset and before the final sleep offset). From these measurements, we calculated total sleep time and sleep efficiency (total sleep time divided by time between lights-out and get-up time, in percentages).

Objective sleep parameters were measured with wrist actigraphy, which consists of a wrist watch-like device that is carried on the non-dominant wrist during the sleep period 
and registers movements. Validation of actigraphy in adolescents with polysomnography showed a sensitivity of 0.95 and a specificity of 0.75 (Meltzer et al. 2012). Participants used the event marker button of the actigraphy to indicate "lights out" and "get-up" times. The actiwatch registered total sleep time, wake after sleep onset, sleep onset latency, and sleep efficiency.

Symptoms of insomnia were measured with the insomnia scale of the Holland Sleep Disorder Questionnaire (HSDQ; Kerkhof et al. 2012). This scale from the HSDQ consists of eight items rated on a five-point Likert scale (1 to 5), with higher scores indicating more severe insomnia symptoms (e.g., "I have difficulty falling asleep" and "I feel sleepy during the day"). Cronbach's alpha was 0.88 in a sample of 951 adolescents from the general population and 210 adolescents from clinical samples (Van Maanen et al. 2014).

Symptoms of chronic sleep reduction were measured with the Chronic Sleep Reduction Questionnaire (CSRQ; Meijer 2008), which consists of 20 items of 3 ordinal response categories (1-3) that measure symptoms of chronic sleep reduction (i.e., shortage of sleep, irritation, loss of energy, and sleepiness) in the previous 2 weeks (e.g., "I am a person who does not get enough sleep," "Others think that I am easily irritated," "I am active during the day," "Do you feel sleepy during the day?"). Higher scores indicate more chronic sleep reduction. Cronbach's alpha ranged between 0.85 and 0.87 in a Dutch and Australian adolescent population (Dewald et al. 2012).

The body scan meditation was introduced in session two of the six treatment sessions. Use of the body scan meditation was measured with a question that was included in the online sleep $\log$ the day before each session (in total four scores for sessions three to six). The question "On average, how often did you practice the body scan exercise in the past week?" was answered with a 3-point ordinal scale on a pull-down menu of the webpage that included the sleep log. Scores ranged from 0 (not al all), 1 (once or a few days), to 2 (every day). Participants were considered to have engaged in the body scan exercise if they scored at least 2 or higher in total, which is at least every day for 1 week, or several days for two or more weeks (i.e., four scores over weeks three to six, range 0-8). In addition, we constructed an average score of application of CBTI techniques that were introduced in all six sessions on a 3-point ordinal scale of 0 (not al all), 1 (once or a few days), to 2 (every day), based on the questions that participants received in their sleep logs on the day before each session (i.e., five scores over weeks two to six, range $0-10$ ).

\section{Data Analyses}

To compare demographic variables between the groups, an independent samples $t$ test and a $\chi^{2}$ test were used. For the outcome variables (actigraphy, sleep logs, and questionnaires), within-group effect sizes were calculated to indicate the degree of change in response to treatment for each group separately. For the main analyses, we used multilevel regression analyses in which repeated measures were nested within participants. All predictor and outcome variables were standardized, so beta coefficients could be interpreted as Cohen's $d$ effect sizes with $0.20,0.50$, and 0.80 indicating small, medium, and large effect sizes, respectively.

\section{Results}

In total 54 adolescents (age $M=15.50$ years, $\mathrm{SD}=1.62,80 \%$ girls) were included in the present study. Based on the scores for practice of the body scan meditation during the treatment, this group was divided in a body scan group (BS) of 26 adolescents (age $M=15.33$ years, $\mathrm{SD}=1.42,77 \%$ girls, average body scan score $=3.04, \mathrm{SD}=1.22$ ) and a non-body scan group (NBS) of 28 adolescents (age $M=15.64$ years, $\mathrm{SD}=$ $1.80,82 \%$ girls, average body scan score $=0.68, \mathrm{SD}=0.48$ ). There were no significant differences between the body scan group and the non-body scan group in age $(t(52)=0.71$, $p>0.05)$ or gender $\left(\chi^{2}(1)=0.23, p>0.05\right)$. Furthermore, to check whether there was a difference in application of exercises from CBTI between the participants in the two groups, the scores of overall application of CBTI techniques were compared between the BS and the NBS groups, and there appeared no significant difference (mean application of CBTI: $\mathrm{BS}=6.33, \mathrm{SD}=1.15, \mathrm{NBS}=6.00, \mathrm{SD}=1.25$, $t(52)=1.01, p>0.05)$.

Table 1 shows all outcomes at each measurement occasion for actigraphy, sleep logs, and questionnaires for both groups.

\section{Results of Treatment for Both Groups}

Results from the multilevel regression analyses (see Table 2) showed significant differences at baseline in subjective sleep onset latency, total sleep time, and sleep efficiency from sleep logs, with better scores for these parameters in the BS group $(\beta=-0.45, p<.001, \beta=0.43, p<.001$, and $\beta=0.34, p<.05$ respectively). None of the other 11 outcome measures showed a significant difference at baseline. After treatment, sleep onset latency and sleep efficiency from actigraphy improved significantly in both groups $(\beta=-$ $0.58, p<.001$, and $\beta=0.62, p<.001$ respectively). All parameters from sleep logs improved for both groups after treatment $(\beta$ 's $=-0.56,-0.38,0.31,0.70$, and 0.26 , all $p$ 's $<.001$, for sleep onset latency, wake after sleep onset, total sleep time, sleep efficiency, and subjective sleep quality respectively). Furthermore, insomnia symptoms measured with the HSDQ decreased significantly for both groups $(\beta=-0.63, p<.001)$, and the symptoms of Loss of energy, Sleepiness, and the total score from the CSRQ decreased significantly for both groups after treatment $(\beta=-0.35$, 
Table 1 Means, standard deviations, and within-group effect sizes (Cohen's $d$ ) of sleep variables from actigraphy, sleep logs, and questionnaires

\begin{tabular}{|c|c|c|c|c|c|}
\hline Sleepparameter & Baseline mean (SD) & Post-treatment mean (SD) & Cohen's $d$ & Follow-up mean (SD) & Cohen's $d$ \\
\hline \multicolumn{6}{|l|}{ Actigraphy } \\
\hline \multicolumn{6}{|l|}{ Body scan group } \\
\hline Sleep onset latency & $38.47(24.76)$ & $21.27(19.20)$ & -0.72 & $15.11(12.93)$ & -1.10 \\
\hline Wake after sleep onset & $81.55(37.23)$ & $71.29(28.02)$ & -0.29 & $65.92(23.35)$ & -0.47 \\
\hline Total sleep time & 423.77 (76.66) & $444.62(60.64)$ & 0.28 & $428.62(67.50)$ & 0.06 \\
\hline Sleep efficiency & $76.09(7.68)$ & $82.86(5.58)$ & 0.94 & $84.24(5.29)$ & 1.15 \\
\hline \multicolumn{6}{|l|}{ Non-body scan group } \\
\hline Sleep onset latency & $33.80(24.05)$ & $20.49(17.78)$ & -0.59 & $18.33(15.87)$ & -0.71 \\
\hline Wake after sleep onset & $73.32(23.67)$ & $72.60(27.68)$ & -0.03 & $74.13(24.40)$ & 0.03 \\
\hline Total sleep time & $405.67(75.02)$ & $412.43(78.82)$ & 0.08 & $410.38(61.56)$ & 0.06 \\
\hline Sleep efficiency & $77.41(6.63)$ & $81.50(5.00)$ & 0.65 & $81.69(4.73)$ & 0.70 \\
\hline \multicolumn{6}{|l|}{ Sleep logs } \\
\hline \multicolumn{6}{|l|}{ Body scan group } \\
\hline Sleep onset latency & $40.80(35.42)$ & $25.94(19.91)$ & -0.48 & $19.57(14.59)$ & -0.73 \\
\hline Wake after sleep onset & $12.23(26.44)$ & $4.06(14.85)$ & -0.35 & $4.83(11.31)$ & -0.34 \\
\hline Total sleep time & $478.79(91.96)$ & $491.32(73.14)$ & 0.14 & $494.81(64.93)$ & 0.19 \\
\hline Sleep efficiency & $85.95(10.11)$ & $91.99(5.63)$ & 0.69 & $93.60(4.56)$ & 0.91 \\
\hline Subjective sleep quality & $2.96(0.98)$ & $3.34(0.86)$ & 0.38 & $3.51(0.92)$ & 0.55 \\
\hline \multicolumn{6}{|l|}{ Non-body scan group } \\
\hline Sleep onset latency & $56.01(45.79)$ & $36.40(29.40)$ & -0.48 & 40.19 (26.79) & -0.39 \\
\hline Wake after sleep onset & $15.88(29.20)$ & $6.99(14.43)$ & -0.36 & $5.68(13.91)$ & -0.42 \\
\hline Total sleep time & $440.92(102.02)$ & $464.84(79.37)$ & 0.24 & $473.71(78.72)$ & 0.34 \\
\hline Sleep efficiency & $82.61(10.20)$ & $88.98(7.78)$ & 0.66 & $88.29(7.08)$ & 0.60 \\
\hline Subjective sleep quality & $2.87(1.01)$ & $3.03(1.14)$ & 0.14 & $3.01(1.04)$ & 0.13 \\
\hline \multicolumn{6}{|l|}{ Questionnaires $^{1}$} \\
\hline \multicolumn{6}{|l|}{ Body scan group } \\
\hline Insomnia symptoms & $3.49(0.53)$ & $2.85(0.76)$ & -0.91 & $2.53(0.66)$ & -1.49 \\
\hline Shortness of sleep & $13.59(2.09)$ & $11.72(2.21)$ & -0.81 & $11.69(1.95)$ & -0.88 \\
\hline Irritation & 9.18 (2.67) & $7.79(2.37)$ & -0.51 & $7.70(1.90)$ & -0.59 \\
\hline Loss of energy & $10.36(2.46)$ & $8.74(2.67)$ & -0.59 & $9.24(2.17)$ & -0.45 \\
\hline Sleepiness & $8.77(2.49)$ & $7.75(2.51)$ & -0.38 & $7.75(1.89)$ & -0.43 \\
\hline Total CSRQ & $41.91(6.86)$ & $36.01(7.36)$ & -0.77 & $36.39(5.62)$ & -0.82 \\
\hline \multicolumn{6}{|l|}{ Non-body scan group } \\
\hline Insomnia symptoms & $3.55(0.59)$ & $3.06(0.72)$ & -0.70 & $2.80(0.86)$ & -0.96 \\
\hline Shortness of sleep & $13.67(2.14)$ & $12.97(2.60)$ & -0.27 & $12.75(2.50)$ & -0.37 \\
\hline Irritation & $8.92(2.98)$ & $8.87(2.54)$ & -0.02 & $8.34(2.34)$ & -0.20 \\
\hline Loss of energy & $10.92(2.57)$ & $10.06(2.31)$ & -0.33 & $9.71(1.89)$ & -0.50 \\
\hline Sleepiness & $8.83(1.86)$ & $8.10(1.55)$ & -0.40 & $7.70(1.69)$ & -0.60 \\
\hline Total CSRQ & $42.33(7.48)$ & $40.00(7.22)$ & -0.30 & $38.49(6.78)$ & -0.50 \\
\hline
\end{tabular}

${ }^{1}$ Insomnia symptoms from the insomnia scale of the Holland Sleep Disorder Questionnaire (Kerkhof et al. 2012); other outcomes from the Chronic Sleep Disorder Questionnaire (CSRQ; Meijer 2008)

$p<.05, \beta=-0.36$, and $\beta=-0.32, p<.05$ respectively). From post-treatment to follow-up, there were no significant changes in any of the outcomes, indicating that all improvements from baseline to post-treatment were maintained, except for symptoms of insomnia, which showed a further decrease for both groups $(\beta=-0.34, p<.05)$.

\section{Differences between the Groups}

The treatment period by group interactions in the multilevel analyses (see Table 2) indicates different effects of treatment for the two groups during the periods from baseline to posttreatment or from post-treatment to follow-up (Fig. 1). There 
Table 2 Parameter estimates from the multilevel regression analyses, for all measurements from actigraphy, sleep logs, and questionnaires

\begin{tabular}{|c|c|c|c|c|c|c|c|c|c|c|c|c|}
\hline \multirow[t]{2}{*}{ Actigraphy } & \multicolumn{2}{|c|}{ Sleep onset latency } & \multicolumn{2}{|c|}{ Wake after sleep onset } & \multicolumn{2}{|c|}{ Total sleep time } & \multicolumn{2}{|c|}{ Sleep efficiency } & & & & \\
\hline & $\beta$ (s.e.) & $p$ & $\beta($ s.e. $)$ & $p$ & $\beta$ (s.e.) & $p$ & $\beta$ (s.e.) & $p$ & & & & \\
\hline $\mathrm{BS}_{\text {vs }} \mathrm{NBS}^{1}$ & $0.24(0.17)$ & .16 & $0.28(0.23)$ & .22 & $0.27(0.15)$ & .07 & $-0.19(0.19)$ & .33 & & & & \\
\hline Post treatment & $-0.58(0.11)$ & $<.001$ & $-0.05(0.10)$ & .61 & $0.12(0.12)$ & .34 & $0.62(0.10)$ & $<.001$ & & & & \\
\hline Follow-up & $-0.11(0.12)$ & .36 & $0.17(0.11)$ & .13 & $0.01(0.13)$ & .93 & $-0.01(0.11)$ & .90 & & & & \\
\hline Group * post treatment & $-0.18(0.15)$ & .23 & $-0.38(0.14)$ & $<.01$ & $0.09(0.17)$ & .61 & $0.37(0.14)$ & $<.01$ & & & & \\
\hline Group * follow-up & $-0.23(0.18)$ & .20 & $-0.33(0.17)$ & $<.05$ & $-0.06(0.20)$ & .78 & $0.31(0.16)$ & $<.05$ & & & & \\
\hline \multirow[t]{2}{*}{ Sleep logs } & \multicolumn{2}{|c|}{ Sleep onset latency } & \multicolumn{2}{|c|}{ Wake after sleep onset } & \multicolumn{2}{|c|}{ Total sleep time } & \multicolumn{2}{|c|}{ Sleep efficiency } & \multicolumn{2}{|c|}{ Subjective sleep quality } & & \\
\hline & $\beta$ (s.e.) & $p$ & $\beta($ s.e. $)$ & $p$ & $\beta$ (s.e.) & $p$ & $\beta($ s.e. $)$ & $p$ & $\beta($ s.e. $)$ & $p$ & & \\
\hline BS vs NBS & $-0.45(0.15)$ & $<.001$ & $-0.11(0.17)$ & .51 & $0.43(0.14)$ & $<.001$ & $0.34(0.15)$ & $<.05$ & $0.07(0.18)$ & .68 & & \\
\hline Post treatment & $-0.56(0.09)$ & $<.001$ & $-0.38(0.10)$ & $<.001$ & $0.31(0.10)$ & $<.001$ & $0.70(0.09)$ & $<.001$ & $0.26(0.09)$ & $<.001$ & & \\
\hline Follow-up & $0.04(0.11)$ & .70 & $-0.04(0.12)$ & .75 & $0.12(0.12)$ & .32 & $-0.03(0.11)$ & .81 & $-0.06(0.10)$ & .55 & & \\
\hline Group * post treatment & $0.14(0.13)$ & .30 & $0.01(0.14)$ & .95 & $-0.17(0.15)$ & .26 & $-0.03(0.13)$ & .81 & $0.13(0.13)$ & .29 & & \\
\hline Group $*$ follow-up & $-0.18(0.16)$ & .26 & $0.14(0.17)$ & .40 & $-0.08(0.18)$ & .65 & $0.14(0.16)$ & .39 & $0.18(0.15)$ & .24 & & \\
\hline \multirow[t]{2}{*}{ Questionnaires } & \multicolumn{2}{|c|}{ Insomnia symptoms } & \multicolumn{2}{|c|}{ Shortness of sleep } & \multicolumn{2}{|c|}{ Irritation } & \multicolumn{2}{|c|}{ Loss of energy } & \multicolumn{2}{|c|}{ Sleepiness } & \multicolumn{2}{|c|}{ Total CSRQ } \\
\hline & $\beta($ s.e.) & $p$ & $\beta($ s.e.) & $p$ & $\beta($ s.e. $)$ & $p$ & $\beta($ s.e. $)$ & $p$ & $\beta($ s.e. $)$ & $p$ & $\beta($ s.e. $)$ & $p$ \\
\hline BS vs NBS & $-0.08(0.26)$ & .76 & $-0.03(0.28)$ & .91 & $0.11(0.29)$ & .71 & $-0.23(0.28)$ & .42 & $-0.03(0.29)$ & .92 & $-0.06(0.28)$ & .83 \\
\hline Post treatment & $-0.63(0.14)$ & $<.001$ & $-0.30(0.16)$ & .07 & $-0.02(0.12)$ & .87 & $-0.35(0.14)$ & $<.05$ & $-0.36(0.15)$ & $<.05$ & $-0.32(0.12)$ & $<.05$ \\
\hline Follow-up & $-0.34(0.14)$ & $<.05$ & $-0.09(0.16)$ & .56 & $-0.21(0.12)$ & .08 & $-0.15(0.14)$ & .31 & $-0.20(0.15)$ & .20 & $-0.21(0.12)$ & .10 \\
\hline Group * post treatment & $-0.19(0.20)$ & .34 & $-0.50(0.23)$ & $<.05$ & $-0.53(0.17)$ & $<.001$ & $-0.32(0.21)$ & .13 & $-0.14(0.22)$ & .52 & $-0.49(0.18)$ & $<.01$ \\
\hline Group $*$ follow-up & $-0.08(0.20)$ & .71 & $0.08(0.23)$ & .73 & $0.18(0.17)$ & .31 & $0.35(0.21)$ & .09 & $0.19(0.22)$ & .37 & $0.26(0.18)$ & 0.15 \\
\hline
\end{tabular}

$B S$, body scan group; $N B S$, non-body scan group; CSRQ, Chronic Sleep Reduction Questionnaire

All outcome variables have been standardized so $\beta$ coefficients can be interpreted as effect sizes with .20, .50, and .80 indicating small, medium, and large effect sizes. Group* post treatment is the interaction of the treatment effect at post treatment for BS compared with NBS group. Group* follow-up is the interaction effect for the (additional) treatment effect from post-treatment to 2 month follow-up of BS compared with NBS. If there is no significant effect at follow-up, the effect at follow-up is similar to the effect at post-treatment

were significant interactions from baseline to post-treatment and from post-treatment to follow-up for wake after sleep onset and sleep efficiency from actigraphy $(\beta=-0.38$, $p<.01, \beta=-0.33, p<.05$ for wake after sleep onset, and $\beta=0.37, p<.01, \beta=0.31, p<.05$ for sleep efficiency, respectively). Furthermore, there were significant interactions from baseline to post treatment for the subscales shortness of sleep and irritation, and for the total score from the CSRQ $(\beta=-$ $0.50, p<.05, \beta=-0.53, p<.001, \beta=-0.49, p<.05$, respectively). All these interactions indicated greater improvement after treatment for the BS group compared with the NBS group. None of the sleep parameters from sleep logs showed significant interactions, nor were there interactions for sleep onset latency and total sleep time from actigraphy, and for insomnia symptoms, loss of energy and sleepiness.

\section{Discussion}

In the present study, we investigated the relation between use of body scan meditation and effectiveness of insomnia treatment for adolescents. Based on self-reported use of a body scan meditation, a group of 54 participants who received internet-delivered CBTI was divided in a body scan group and a non-body scan group, and compared with subjective and objective sleep outcomes from baseline to posttreatment and to 2-month follow-up. Results showed that both groups improved with small to large effect sizes at post treatment for most of the subjective and objective outcome measures for sleep and symptoms of insomnia and chronic sleep reduction and that improvements were maintained at follow-up. However, where the non-body scan group showed no significant change of wake after sleep onset from actigraphy, and shortness of sleep and irritation from the CSRQ, there were medium effect size improvements of these outcomes in the body scan group. These results indicate that the use of a body scan meditation in CBTI may have an additional positive effect on sleep, above and beyond the effect of the traditional CBTI techniques.

Most of the results from this study confirm the preliminary efficacy of CBTI in adolescents that has been found in other studies, with improvements of sleep variables from both objective and subjective measurements. However, where up until now no studies with adolescents have indicated large effects of CBTI on objective wake after sleep onset, this study indicated a differential effect of treatment on this measure between adolescents that did or did not engage in a body scan meditation. This is especially striking since previous research has been unable to fully explain the substantially larger amounts of wake after sleep onset measured with actigraphy compared with the subjectively reported small amounts of wake after

Fig. 1 Change from baseline to post-treatment and 2-month follow-up of $\mathrm{z}$ scores for wake after sleep onset from actigraphy and shortness of sleep and irritation from the chronic sleep reduction questionnaire, for adolescents who did or did not use the body scan meditation exercise during insomnia treatment 


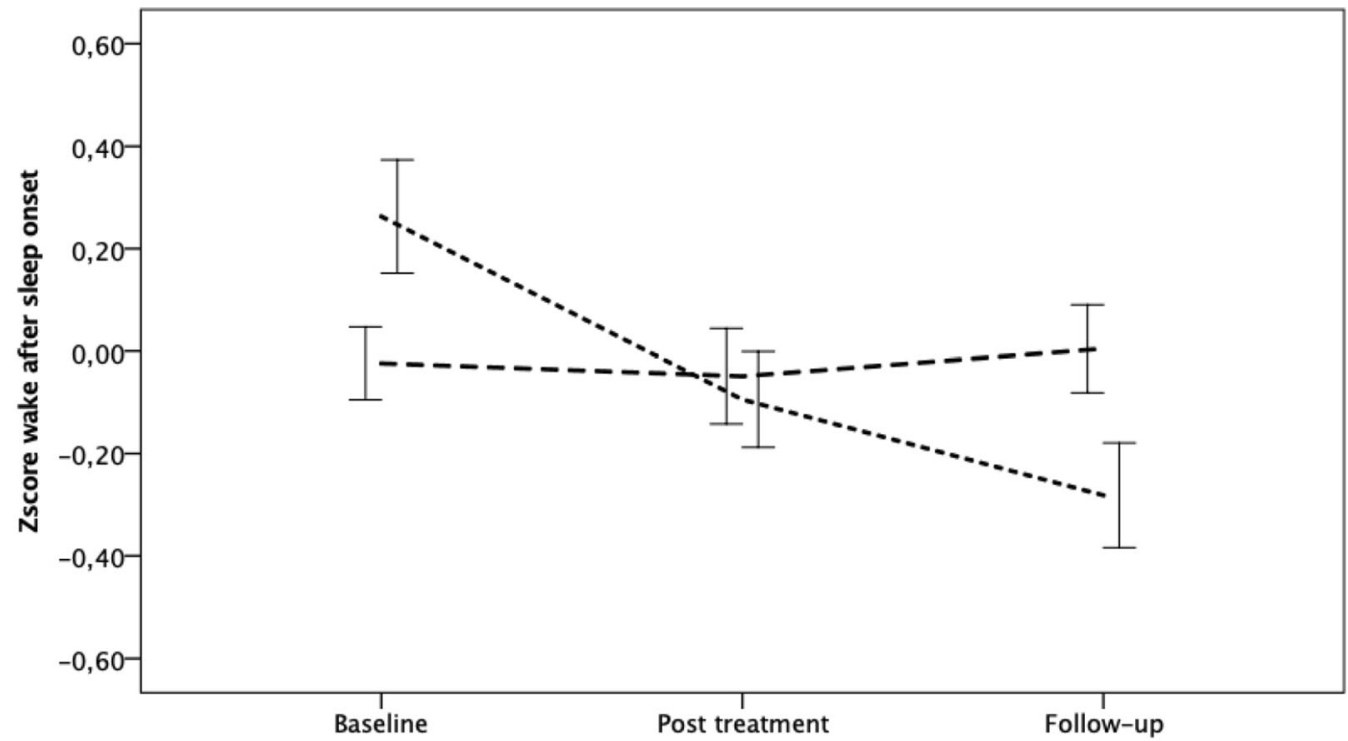

- - Non body scan group --- Body scan group

Error bars: +/- $1 \mathrm{SE}$
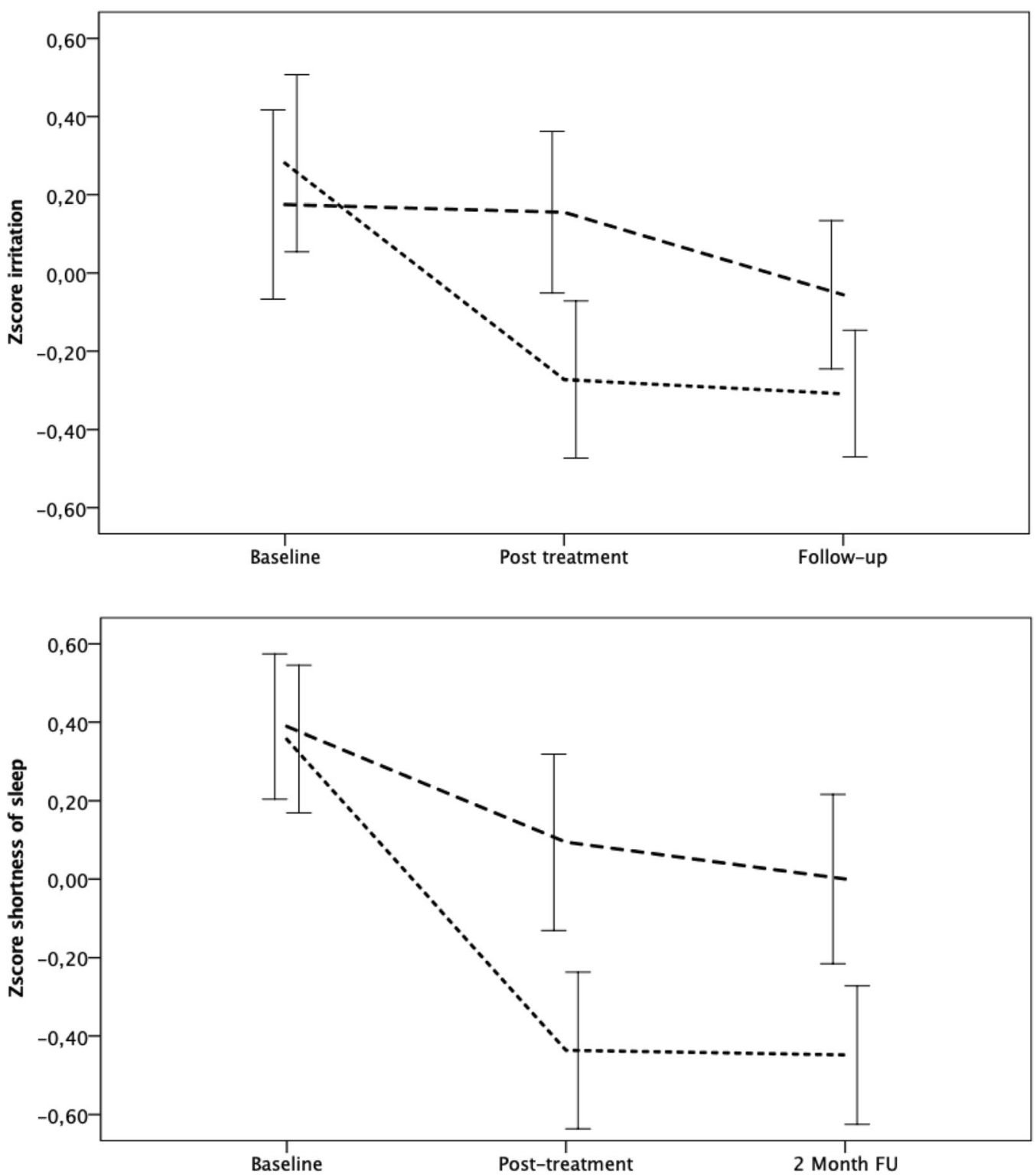
sleep onset in sleep logs. Authors have suggested that this difference is due to naturally occurring high levels of sleep motor activity in adolescents compared with adults, which is coded as wake by actigraphy (Short et al. 2012). In other words, adolescents simply move more while asleep. The subjective reporting of short wake after sleep onset in sleep logs by adolescents would confirm these suggestions, but the present study shows that in adolescents with insomnia, objective wake after sleep onset can decrease after treatment that includes a body scan meditation. This points to an insomnia factor that goes mostly undetected by adolescents themselves and is not fully addressed by traditional CBTI techniques. A similar pattern of improvements for the body scan group but not for the non-body scan group was found for the scales shortness of sleep and irritation, and the total score, from the chronic sleep reduction questionnaire. The subscale shortness of sleep measures a concept in which the amount of sleep is limited by either restriction of sleep or a diminished sleep quality or increased fragmentation. The subscale irritation measures feelings of irritability that can result from inadequate sleep over a longer period of time. The concurrent differential improvements for shortness of sleep, irritation, and objective wake after sleep onset in adolescents who did or did not engage in the body scan meditation could be caused by a shared underlying factor, such as increased levels of psychophysiological arousal because of excessive thoughts, worry, or rumination, which is specifically affected by the body scan meditation and the non-judgmental awareness it promotes. However, the absence of subjective reports of excessive wake after sleep onset and the lack of its decrease after treatment could indicate that this is not a clinically meaningful aspect of sleep for adolescents, but the concurrent decrease of shortness of sleep and irritation does indicate that it has an effect on adolescents' daily life and well-being. As optimal cutoff for the total CSRQ score, 39.5 was calculated (DewaldKaufmann, et al., 2018), and in both groups the mean scores decreased to below that cutoff, with the largest decrease in the body scan group. This could indicate a clinically significant improvement for many adolescents in our study. Further research with suitable methods to unravel these issues is needed.

At baseline, the body scan group had a subjectively shorter sleep onset latency, with concurrent higher total sleep time and sleep efficiency, compared with the non-body scan group, but both groups reported a similar medium effect size decrease of SOL after treatment. This effect of CBTI on sleep onset latency was in line with the outcomes from actigraphy, and there were no differential effects for the two groups. Regarding the question whether the body scan group, due to a possibly higher severity of insomnia symptoms, simply practiced any or all techniques of CBTI more, compared with the non-body scan group, our results indicated that this was not the case. There was no difference between the groups in overall use of CBTI techniques.
In general, adolescents with insomnia experience problems predominantly with initiating sleep, as indicated by measures of long sleep onset latency, whereas adults with insomnia have more problems with maintaining sleep, as seen in higher levels of wake after sleep onset (Gradisar et al. 2011). This indicates that the effect of the body scan meditation was specific to wake after sleep onset, shortness of sleep and irritation only, but not to sleep onset latency. This is in line with research from Bartel et al. (2018) who found no decrease in sleep onset latency after a school-based intervention for adolescents that included a mindfulness body scan exercise to use around bedtime aimed at reducing sleep onset latency. Only in adolescents with prolonged sleep onset latency, an effect of the intervention was observed, compared with constructive worry or a control condition. The authors did not include wake after sleep onset in their measurements. These findings imply that the body scan meditation specifically affects sleep problems that occur during the sleep period, such as fragmentation or sleep quality. The mechanism by which the body scan meditation specifically affects wake after sleep onset in adolescents is unclear. Both biological and cognitivebehavioral perspectives could offer plausible factors that are influenced by the body scan meditation, such as the polyvagal theory in which specific breathing and meditative practices trigger physiological states that can improve sleep quality (Porges 2017; Tsai et al. 2015) and the psychobiological inhibition model of insomnia in which selective attending to life stressors and insomnia symptoms results in sleep effort and perpetuates insomnia (Espie et al. 2006). However, further studies are needed to investigate these assumptions.

The efficacy of CBTI in adolescents has only recently been studied in a randomized controlled design, which showed that it is effective in both internet- and group-therapy modalities and that effects are comparable to adult CBTI (De Bruin et al., 2015, De Bruin et al., 2018). However, CBTI techniques are derived from models of insomnia that are based on adults. It is yet unclear whether these models, and the corresponding techniques of CBTI, fully cover the factors that cause or perpetuate behavioral sleep problems such as insomnia in adolescents. Furthermore, since cognitive therapy as part of CBTI is aimed at changing dysfunctional thoughts, whereas mindfulnessbased techniques such as the body scan focus on acceptance, it would be interesting to see the differential effects of these techniques in a direct comparison. These issues were beyond the scope of the present study, and more research in these areas of adolescent sleep problems is needed.

\section{Limitations and Future Research}

Although this study included a relatively novel technique for the treatment of insomnia in adolescents, namely the body scan, in combination with traditional CBTI techniques, and the study was conducted in a longitudinal design with both objective and subjective outcome measurements, there are 
also some limitations. First of all, participants in the BS and NBS groups were not randomly assigned to these conditions. This implies that the differential treatment effects that we found might be caused by an underlying confounding factor, such as motivation to engage in all the treatment techniques. However, from the measures of engagement in the traditional CBTI techniques, there appeared no difference between the groups, which would imply that both groups engaged similarly in the treatment. Furthermore, we found the differential effects between the groups in an objective measurement of WASO, which subjectively goes mostly undetected. If a confounding factor were in play, it would still point to a previously unaddressed aspect of insomnia that in our application of CBTI was affected. Additional to motivation, the results could also be influenced by the willingness of adolescents to engage in a mindfulness intervention or other modules in the treatment. Although this limits the generalizability of our results, it could also point to a factor on which individual tailoring of CBTI could be based. If effects of CBTI are increased for adolescents who are willing to use mindfulness meditation, then tailoring to include this technique in their specific individual treatment would be appropriate. Whether and how this willingness to engage in mindfulness meditations has a differential influence on effectiveness of CBTI in adolescents needs further research. Also, more studies are needed to investigate which aspects of sleep and insomnia are affected by meditation practices, preferably in an experimental setting, using additional objective measurements such as polysomnography. Another limitation is that we studied the effects of CBTI in an internet setting only. The use of a mindfulness body scan meditation could be different in a group setting where during the group sessions, every participant could practice that exercise several times in the six sessions. If in internet therapy, benefits from the body scan meditation are not immediately noticeable; participants could be reluctant to practice the exercise after its initial introduction. However, this is not different from the application of other therapeutic techniques in internet therapy, and the results from this study do indicate that the online application of a mindfulness exercise for the treatment of insomnia in adolescents is feasible. Further studies in a randomized controlled design are warranted.

Author Contributions EdB: designed and executed the study and the data analyses, and wrote the paper. AMM: collaborated with the design, the analyses of the data, and writing of the study. SB: collaborated with the design and writing of the study. All authors approved the final version of the manuscript for submission.

\section{Compliance with Ethical Standards}

Conflict of Interests The authors declare that they have no conflict of interest.
Ethical Approval All procedures performed in this study were in accordance with the ethical standards of the institutional and/or national research committee and with the 1964 Helsinki declaration and its later amendments or comparable ethical standards. This study received ethical approval by the medical ethical committee of the Academic Medical Center in Amsterdam and is registered at the International Standard Randomized Controlled Trial Number with the number ISRCTN33922163.

Informed Consent Informed consent was obtained from all individual participants included in this study.

Open Access This article is licensed under a Creative Commons Attribution 4.0 International License, which permits use, sharing, adaptation, distribution and reproduction in any medium or format, as long as you give appropriate credit to the original author(s) and the source, provide a link to the Creative Commons licence, and indicate if changes were made. The images or other third party material in this article are included in the article's Creative Commons licence, unless indicated otherwise in a credit line to the material. If material is not included in the article's Creative Commons licence and your intended use is not permitted by statutory regulation or exceeds the permitted use, you will need to obtain permission directly from the copyright holder. To view a copy of this licence, visit http://creativecommons.org/licenses/by/4.0/.

\section{References}

Achenbach, T. M. (1991). Manual for the youth self-report and 1991 profile. Burlington, VT: Department of Psychiatry, University of Vermont.

American Psychiatric Association. (2013). Diagnostic and statistical manual of mental disorders: DSM-5 (5th ed.). Washington, D.C.: Author

Bartel, K., Huang, C., Maddock, B., Williamson, P., \& Gradisar, M. (2018). Brief school-based interventions to assist adolescents' sleep-onset latency: comparing mindfulness and constructive worry versus controls. Journal of Sleep Research, 27(3), e12668.

Blake, M., Waloszek, J., Schwartz, O., Raniti, M., Simmons, J., Blake, L., et al. (2016). The SENSE study: post intervention effects of a randomized controlled trial of a cognitive-behavioral and mindfulnessbased group sleep improvement intervention among at-risk adolescents. Journal of Consulting and Clinical Psychology, 84(12), 1039-1051.

Bonnet, M. H., \& Arand, D. L. (2010). Hyperarousal and insomnia: state of the science. Sleep Medicine Reviews, 14(1), 9-15.

Bootzin, R. R., \& Stevens, S. J. (2005). Adolescents, substance abuse, and the treatment of insomnia and daytime sleepiness. Clinical Psychology Reviews, 25(5), 629-644. https://doi.org/10.1016/j.cpr. 2005.04.007.

Chervin, R. D., Dillon, J. E., Bassetti, C., Ganoczy, D. A., \& Pituch, K. J. (1997). Symptoms of sleep disorders, inattention, and hyperactivity in children. Sleep, 20(12), 1185-1192.

Dahl, R. E., \& Lewin, D. S. (2002). Pathways to adolescent health sleep regulation and behavior. Journal of Adolescent Health, 31 (6 Suppl), 175-184.

De Bruin, E. J., Bögels, S. M., Oort, F. J., \& Meijer, A. M. (2015a). Efficacy of cognitive behavioral therapy for insomnia in adolescents: a randomized controlled trial with internet therapy, group therapy and a waiting list condition. Sleep, 38, 1913-1926.

De Bruin, E. J., Bögels, S. M., Oort, F. J., \& Meijer, A. M. (2018). Improvements of adolescent psychopathology after insomnia treatment: results from a randomized controlled trial over 1 year. Journal 
of Child Psychology and Psychiatry., 59(5), 509-522. https://doi. org/10.1111/jcpp.12834.

De Bruin, E. J., Dewald-Kaufmann, J. F., Oort, F. J., Bögels, S. M., \& Meijer, A. M. (2015b). Differential effects of online insomnia treatment on executive functions in adolescents. Sleep Medicine, 16(4), 510-520. https://doi.org/10.1016/j.sleep.2014.12.009.

De Bruin, E. J., Van Kampen, R. K. A., Van Kooten, T., \& Meijer, A. M. (2014b). Psychometric properties and clinical relevance of the adolescent sleep hygiene scale in Dutch adolescents. Sleep Medicine, 15, 789-797. https://doi.org/10.1016/j.sleep.2014.03.015.

De Bruin, E., Oort, F., Bögels, S., \& Meijer, A. (2014a). Efficacy of internet and group-administered cognitive behavioral therapy for insomnia in adolescents: a pilot study. Behavioral Sleep Medicine, 12(3), 235-254.

Dewald-Kaufmann, J. F., De Bruin, E. J., Smits, M., Zijlstra, B. J. H., Oort, F. J., \& Meijer, A. M. (2018). Chronic sleep reduction in adolescents-clinical cut-off scores for the chronic sleep reduction questionnaire (CSRQ). Journal of Sleep Research, 27, 1-4. https:// doi.org/10.1111/jsr.12653.

Dewald, J. F., Short, M. A., Gradisar, M., Oort, F. J., \& Meijer, A. M. (2012). The chronic sleep reduction questionnaire (CSRQ): a crosscultural comparison and validation in Dutch and Australian adolescents. Journal of Sleep Research, 21, 584-594.

Dohnt, H., Gradisar, M., \& Short, M. A. (2012). Insomnia and its symptoms in adolescents: comparing DSM-IV and ICSD-II diagnostic criteria. Journal of Clinical Sleep Medicine, 8(3), 295-299. https:// doi.org/10.5664/jcsm.1918.

Espie, C. A., Kyle, S. D., Williams, C., et al. (2012). A randomised, placebo-controlled trial of online cognitive behavioural therapy for chronic insomnia disorder delivered via an automated media-rich web application. Sleep, 35, 769e81.

Espie, C. A., Broomfield, N. M., MacMahon, K. M. A., Macphee, L. M., \& Taylor, L. M. (2006). The attention-intention-effort pathway in the development of psychophysiologic insomnia: a theoretical review. Sleep Medicine Reviews, 10(4), 215-245.

Fernandez-Mendoza, J., Li, Y., Vgontzas, A. N., Fang, J., Gaines, J., Calhoun, S. L., et al. (2016). Insomnia is associated with cortical hyperarousal as early as adolescence. Sleep, 39(5), 1029-1036. https://doi.org/10.5665/sleep.5746.

Gradisar, M., Gardner, G., \& Dohnt, H. (2011). Recent worldwide sleep patterns and problems during adolescence: a review and metaanalysis of age, region, and sleep. Sleep Medicine, 12(2), 110-118.

Gregory, A., \& Sadeh, A. (2012). Sleep, emotional and behavioral difficulties in children and adolescents. Sleep Medicine Reviews, 16(2), 129-136.

Hauri, P., \& Fischer, J. (1986). Persistent psychophysiological (learned) insomnia. Sleep, 9, 38-53.

Hysing, M., Pallesen, S., Stormark, K. M., Lundervold, A. J., \& Sivertsen, B. (2013). Sleep patterns and insomnia among adolescents: a population-based study. Journal of Sleep Research, 22(5), 549-556. https://doi.org/10.1111/jsr.12055.

Johnson, E. O., Roth, T., \& Breslau, N. (2006). The association of insomnia with anxiety disorders and depression: exploration of the direction of risk. Journal of Psychiatric Research, 40(8), 700-708. https://doi.org/10.1016/j.jpsychires.2006.07.008.

Kabat-Zinn, J. (2016). Lying down meditations. Mindfulness, 7(6), 1437 1440.

Kahn, M., Sheppes, G., \& Sadeh, A. (2013). Sleep and emotions: Bidirectional links and underlying mechanisms. International Journal of Psychophysiology, 89(2), 218-228.

Lancee, J., van den Bout, J., van Straten, A., et al. (2012) Internetdelivered or mailed selfhelp treatment for insomnia? A randomized waiting-list controlled trial. Behaviour Research and Therapy, 50(1), 22e9. https://doi.org/10.1016/j.brat.2011.09.012 .

Medalie, L. S., \& Cifu, A. (2017). Management of chronic insomnia disorder in adults. Journal of the American Medical Association, $317,762-763$.

Meijer, A. (2008). Chronic sleep reduction, functioning at school and school performance in adolescents. Journal of Sleep Research, 17, 395-405.

Meijer, A. M., Reitz, E., Dekovic, M., van den Wittenboer, G. L., \& Stoel, R. D. (2010). Longitudinal relations between sleep quality, time in bed and adolescent problem behaviour. Journal of Child Psychology and Psychiatry, 51(11), 1278-1286. https://doi.org/10.1111/j.14697610.2010.02261.x.

Meltzer, L. J., Montgomery-Downs, H. E., Insana, S. P., \& Walsh, C. M. (2012). Use of actigraphy for assessment in pediatric sleep research. Sleep Medicine Reviews, 16(5), 463-475.

Morgenthaler, T., Kramer, M., Alessi, C., Friedman, L., Boehlecke, B., Brown, T., et al. (2006). Practice parameters for the psychological and behavioral treatment of insomnia: an update. An american academy of sleep medicine report. Sleep, 29(11), 1415-1419.

Morin, C. M., Bootzin, R. R., Buysse, D. J., Edinger, J. D., \& Lichstein, K. L. (2006). Psychological and behavioral treatment of insomnia: update of the recent evidence (1998-2004). Sleep, 29(11), 1398e414.

Ong, J., Manber, R., Segal, Z., Xia, Y., Shapiro, S., \& Wyatt, J. (2014). A randomized controlled trial of mindfulness meditation for chronic insomnia. Sleep, 37(9), 1553-1563.

Owens, J., Gruber, R., Brown, T., Corkum, P., Cortese, S., O’Brien, L., et al. (2013). Future research directions in sleep and ADHD: report of a consensus working group. Journal of Attention Disorders, 17(7), 550-564. https://doi.org/10.1177/1087054712457992.

Palagini, L., Ong, J., \& Riemann, D. (2017). The mediating role of sleeprelated metacognitive processes in trait and pre-sleep state hyperarousal in insomnia disorder. Journal of Psychosomatic Research, 99, 59-65.

Perlis, M., Giles, D., Mendelson, W., Bootzin, R., \& Wyatt, J. (1997). Psychophysiological insomnia: the behavioural model and a neurocognitive perspective. Journal of Sleep Research, 6(3), 179188.

Perlis, M. J., Shaw, P. A., Cano, G., \& Espie, C. (2010). Models of insomnia. In M. Kryger, T. Roth, and W. Dement (Eds.), Principles and Practice of Sleep Medicine (5th ed.; pp. 850-865). Elsevier.

Polanczyk, G. V., Salum, G. A., Sugaya, L. S., Caye, A., \& Rohde, L. A. (2015). Annual research review: a meta-analysis of the worldwide prevalence of mental disorders in children and adolescents. Journal of Child Psychology and Psychiatry, 56(3), 345-365. https://doi.org/ 10.1111/jcpp.12381.

Porges, S. (2017). Vagal pathways: portals to compassion. In The Oxford Handbook of Compassion Science. Oxford University Press.

Riemann, D., Spiegelhalder, K., Feige, B., Voderholzer, U., Berger, M., Perlis, M., \& Nissen, C. (2010). The hyperarousal model of insomnia: a review of the concept and its evidence. Sleep Medicine Reviews, 14(1), 19-31.

Roberts, R. E., \& Duong, H. T. (2013). Depression and insomnia among adolescents: a prospective perspective. Journal of Affective Disorders, 148(1), 66-71. https://doi.org/10.1016/j.jad.2012.11. 049.

Sivertsen, B., Salo, P., Mykletun, A., Hysing, M., Pallesen, S., Krokstad, S., et al. (2012). The bidirectional association between depression and insomnia: the HUNT study. Psychosomatic Medicine, 74(7), $758-765$. 
Short, M. A., Gradisar, M., Lack, L. C., Wright, H., \& Carskadon, M. A. (2012). The discrepancy between actigraphic and sleep diary measures of sleep in adolescents. Sleep Medicine, 13, 378-384.

Spielman, A., Caruso, L., \& Glovinsky, P. (1987). A behavioral perspective on insomnia treatment. The Psychiatric Clinics of North America, 10, 541-553.

Tsai, H., Kuo, T., Lee, G., \& Yang, C. (2015). Efficacy of paced breathing for insomnia: enhances vagal activity and improves sleep quality. Psychophysiology, 52(3), 388-396.

Van Maanen, A., Dewald-Kaufmann, J. F., Oort, F. J., de Bruin, E. J., Smits, M. G., Short, M. A., et al. (2014). Screening for sleep reduction in adolescents through self-report: development and validation of the sleep reduction screening questionnaire (SRSQ). Child \& Youth Care Forum, 43(5), 607-619.

Van Straten, A., Van Der Zweerde, T., Kleiboer, A., Cuijpers, P., Morin, C. M., \& Lancee, J. (2018). Cognitive and behavioral therapies in the treatment of insomnia: a meta-analysis. Sleep Medicine Reviews, $38,3-16$.
Wittchen, H. U., Jacobi, F., Rehm, J., Gustavsson, A., Svensson, M., Jönsson, B., et al. (2011). The size and burden of mental disorders and other disorders of the brain in Europe 2010. European Neuropsychopharmacology, 21(9), 655-679.

Wong, S. Y., Zhang, D., Li, C. C., Yip, B. H., Chan, D. C., Ling, Y., Lo, C. S., Woo, D. M., Sun, Y., ... Wing, Y. (2017). Comparing the effects of mindfulness-based cognitive therapy and sleep psycho-education with exercise on chronic insomnia: a randomised controlled trial. Psychotherapy and Psychosomatics, 86(4), 241-253.

Zhang, J., Lam, S. P., Li, S. X., Li, A. M., Lai, K. Y., \& Wing, Y. K. (2011). Longitudinal course and outcome of chronic insomnia in Hong Kong Chinese children: a 5-year follow-up study of a community-based cohort. Sleep, 34(10), 1395-1402. https://doi. org/10.5665/SLEEP.1286.

Publisher's Note Springer Nature remains neutral with regard to jurisdictional claims in published maps and institutional affiliations. 